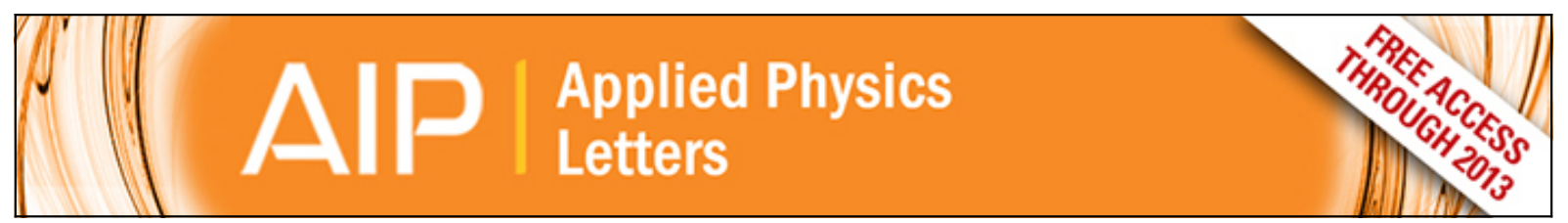

Compositional study of defects in microcrystalline silicon solar cells using spectral decomposition in the scanning transmission electron microscope

M. Duchamp, M. Lachmann, C. B. Boothroyd, A. Kovács, F.-J. Haug, C. Ballif, and R. E. Dunin-Borkowski

Citation: Applied Physics Letters 102, 133902 (2013); doi: 10.1063/1.4800569

View online: http://dx.doi.org/10.1063/1.4800569

View Table of Contents: http://scitation.aip.org/content/aip/journal/apl/102/13?ver=pdfcov

Published by the AIP Publishing

$\underset{\substack{\text { Publoning } \\ \text { PIP }}}{A}$ Re-register for Table of Content Alerts 


\title{
Compositional study of defects in microcrystalline silicon solar cells using spectral decomposition in the scanning transmission electron microscope
}

\author{
M. Duchamp, ${ }^{1, a)}$ M. Lachmann, ${ }^{1}$ C. B. Boothroyd,${ }^{1}$ A. Kovács, ${ }^{1}$ F.-J. Haug, ${ }^{2}$ C. Ballif, ${ }^{2}$ \\ and R. E. Dunin-Borkowski ${ }^{1}$ \\ ${ }^{1}$ Ernst Ruska-Centre for Microscopy and Spectroscopy with Electrons (ER-C) and Peter Grünberg Institute \\ (PGI), Forschungszentrum Jülich, D-52425 Jülich, Germany \\ ${ }^{2}$ Ecole Polytechnique Fédérale de Lausanne (EPFL), Institute of Microengineering (IMT), \\ Photovoltaics and Thin Film Electronics Laboratory, CH-2000 Neuchâtel, Switzerland
}

(Received 18 February 2013; accepted 25 March 2013; published online 5 April 2013)

\begin{abstract}
The chemical compositions of defective regions in microcrystalline thin film $\mathrm{Si}$ solar cells are studied using energy-dispersive X-ray spectroscopy and electron energy-loss spectroscopy (EELS) in the scanning transmission electron microscope. Nanometer-resolved chemical analysis reveals the presence of $\mathrm{ZnO}$ in micrometer-long defective regions. Due to the recent application of unmixing algorithm to EELS, the chemical compositions of the defective regions are determined objectively, without introducing artefacts from the fitting procedures. It is shown that the defective regions in the $\mathrm{Si}$ layer are filled by $\mathrm{ZnO}$, which diffuses along voids that propagate from the bottom up to the top $\mathrm{ZnO}$ contacts. @ 2013 American Institute of Physics. [http://dx.doi.org/10.1063/1.4800569]
\end{abstract}

Thin film Si solar cells require additional elements to enhance light absorption because $\mathrm{Si}$ is a poor light absorber, converting only a fraction of the incident light directly into electron-hole pairs. Such enhancement is normally achieved by incorporating random ${ }^{1,2}$ or periodic ${ }^{3,4}$ substrate interface textures in order to scatter light. However, the use of a highly textured substrate can result in the formation of defective regions above V-shaped valleys, which can extend through the entire active Si layer. ${ }^{5,6}$ Recently, focused ion beam (FIB) "slice and view" tomography has been used to show that such defects can take the form of continuous networks of filaments of defective material, rather than simple line defects that extend between the top and bottom contacts. ${ }^{7}$ Although the deleterious effect of the defective regions on the electrical properties of solar cells can be limited by smoothing the texture of the electrode on which the active layers are deposited, ${ }^{7}$ textures with high aspect ratios are needed for strong light scattering in order to achieve an increase of up to $45 \%$ in current density. ${ }^{8}$

Scanning transmission electron microscopy (STEM) allows direct imaging of the chemical compositions of materials. High-angle annular dark-field (HAADF) images, which are sensitive to local variations in atomic number and specimen thickness, can be recorded at the same time as energydispersive X-ray (EDX) and electron-energy-loss (EEL) spectra. The latter signals can be used to provide compositional information with close-to-atomic spatial resolution. ${ }^{9,10}$ Furthermore, chemical bonding information can be obtained from energy-loss near-edge structure $\left(\right.$ ELNES) ${ }^{11}$ and pixel-by-pixel elemental maps can be determined from the recorded spectra. ${ }^{12}$

In this letter, the elemental compositions of defective regions in a hydrogenated microcrystalline $\mathrm{Si}(\mu \mathrm{c}-\mathrm{Si}: \mathrm{H})$ solar cell are determined on the nanometer scale. In particular, we apply spectral unmixing (SU) using a vortex component analysis (VCA) algorithm, which has only recently been

a)Tel.: +49 246161 9478. Electronic mail: martial.duchamp@gmail.com. applied to electron energy-loss spectroscopy (EELS) spectra, to determine local chemical compositions and to determine bonding information from recorded EEL spectra. ${ }^{12}$

Experimental results were obtained from a singlejunction $n-i-p \mu \mathrm{c}-\mathrm{Si}: \mathrm{H}$ solar cell grown on a glass substrate. The bottom (back) electrode consists of a 2- $\mu$ m-thick B-doped $\mathrm{ZnO}$ layer, which was deposited using low-pressure chemical vapour deposition (LP-CVD) and has a V-shaped texture. The active $2-\mu \mathrm{m}$-thick $\mu \mathrm{c}$-Si:H layers were deposited using plasma-enhanced chemical vapour deposition (PE-CVD). No smoothing was applied to the textured $\mathrm{ZnO}$ layer. The top (front) contact was also a $2-\mu$ m-thick B-doped $\mathrm{ZnO}$ layer deposited using LP-CVD.

An 80-nm-thick electron-transparent TEM specimen was prepared using a dual-beam FIB workstation. STEM EDX mapping was carried out using an EDAX Si(Li) detector on an FEI Tecnai F20 microscope operated at $200 \mathrm{kV}$. A post-column Gatan Imaging Filter (GIF Quantum) was used for STEM EEL spectrum imaging on a double- $\mathrm{C}_{\mathrm{S}}$-corrected FEI Titan microscope operated at $300 \mathrm{kV}$. EDX spectrum images were $15 \times 50 \times 4096$ pixels in size, corresponding to dimensions of $150 \mathrm{~nm} \times 500 \mathrm{~nm} \times 20.48 \mathrm{keV}$ and were recorded using an acquisition time of $1 \mathrm{~s} /$ pixel at $5 \mathrm{eV} / \mathrm{chan}-$ nel. EEL spectra were recorded on a 2048 pixel camera in image-coupled mode using a convergence semi-angle of 25 mrad and a collection semi-angle of $\sim 25 \mathrm{mrad}$ with a size of $51 \times 218 \times 2048$ pixels, corresponding to dimensions of $0.31 \mu \mathrm{m} \times 1.34 \mu \mathrm{m} \times 2048 \mathrm{eV}$, using an acquisition time of $1 \mathrm{~s} /$ pixel at $1 \mathrm{eV} /$ channel.

Figure 1(a) shows an HAADF STEM image of the specimen, in which a rectangle indicates a defective region, which propagates from the bottom of the $\mathrm{ZnO}$ layer into the active $\mu \mathrm{c}-\mathrm{Si}: \mathrm{H}$ and was chosen for detailed examination below. In this image, darker contrast corresponds to regions that have a lower atomic number or density. Figures 1(b)-1(d) are elemental maps obtained by integrating the (b) Si K, (c) O K, and (d) $\mathrm{Zn} \mathrm{K} \mathrm{K}_{\alpha}$ EDX peak intensities. Maps obtained using the $\mathrm{Zn} \mathrm{K} \beta$ and $\mathrm{Zn} \mathrm{L}_{\alpha}$ edges (not shown) were similar to Fig. 1(d). 


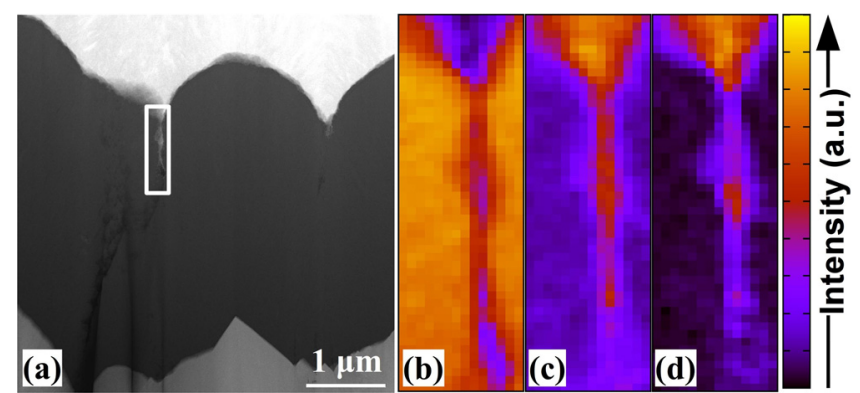

FIG. 1. (a) HAADF STEM image of an FIB-prepared TEM specimen of a solar cell. The white rectangle indicates an area close to the front contact, which was analysed in detail using EDX and EEL spectroscopy. (b)-(d) EDX maps obtained using the (b) Si K, (c) O K, and (d) $\mathrm{Zn} \mathrm{K}_{\alpha}$ signals. The field of view for (b)-(d) is $150 \mathrm{~nm} \times 500 \mathrm{~nm}$.

The highest number of counts was $\sim 40$ for $\mathrm{Zn} \mathrm{K}_{\alpha}$ and $\sim 70$ for Si K. As the background level below the peaks was below 2 counts and the peaks of interest did not overlap, direct integration of the peak areas was used to obtain the maps. Some of the Si signal in the defective region may originate from the surrounding Si matrix. ${ }^{7}$ Whereas the $\mathrm{Zn}$ signal is confined to the defective area, it is impossible to use the EDX maps to establish whether the $\mathrm{O}$ signal is associated with surface oxidation of the TEM specimen or if it originates from the growth of the solar cell. This ambiguity is now addressed by using EELS combined with SU using VCA.

Figure 2(a) shows an HAADF STEM image recorded pixel-by-pixel together with EEL spectra from the same region as in Fig. 1. The resulting background-subtracted Si L, C K, O K, Zn L and Si K EEL signals are shown in Figs. 2(b)-2(f). The presence of $\mathrm{O}$ and $\mathrm{Zn}$ in the defective regions can be seen in Figs. 2(d) and 2(e), respectively. The $\mathrm{Zn}$ signal appears to decrease towards the lower part of this region, whereas the $\mathrm{C}$ signal appears to increase, as shown in Fig. 2(c). The presence of $\mathrm{C}$ is likely to be associated with post-specimen-preparation contamination. The apparent substitution of $\mathrm{Zn}$ by $\mathrm{C}$ may, therefore, be explained by the

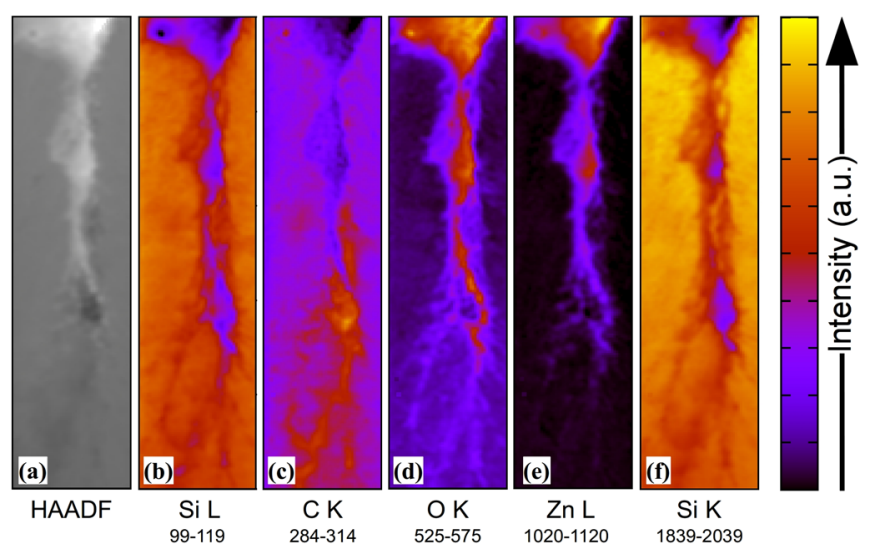

FIG. 2. (a) High-angle annular dark-field STEM image and (b)-(f) background-subtracted EEL spectrum images of a defective region in the region of the solar cell indicated in Fig. 1. The maps were obtained on a pixelby-pixel basis from the recorded 3D dataset by subtracting a power-law background as a function of energy beneath each edge and integrating the resulting edge intensity. The field of view in each figure is $0.31 \mu \mathrm{m} \times 1.34 \mu \mathrm{m}$. The integration ranges in $\mathrm{eV}$ are indicated below each map. presence of voids in the interior of the defective region in the Si layer, which are also less accessible for the diffusion of Zn during growth. Although the signal collected at the Si K edge is two orders of magnitude lower than that at the Si L edge, it is affected much less by the presence of preceding edges. The $\mathrm{O}$ signal shows a strong correlation with the $\mathrm{Zn}$ signal. However, $\mathrm{O}$ is also present in the $\mathrm{Si}$-rich regions, suggesting that some of the $\mathrm{O}$ signal may be associated with the presence of $\mathrm{O}$ on the surfaces of the TEM specimen.

An important advantage of using EEL over EDX spectroscopy is its sensitivity to chemical states and to the local atomic environment in the specimen. In particular, ELNES can be compared with that from known materials and used to identify the local atomic coordination of each element. A number of different statistical methods can be used to separate the different contributions to recorded EEL edge shapes from a large dataset into components. ${ }^{13,14}$ Recently, SU has been proposed for the analysis of EEL spectrum images and shown to be superior to the use of principal or independent component analysis. ${ }^{12} \mathrm{SU}$ allows signals that have poor signal-tonoise ratio but can be obtained in the form of an average of a given spectral component within an area of interest to be extracted. In this way, not only elemental maps but also "chemical bonding" maps can be obtained using spectrum imaging. In order to determine the chemical state of each element in the defective region in the present specimen, SU was applied by performing VCA using an algorithm provided by Nascimento et al., ${ }^{15}$ run under GNU OCTAVE software.

When using this method, the number of components to be extracted from an EEL spectrum image must be chosen by the user. In our first attempt, we assumed that the main components would be associated with the presence of $\mathrm{Si}, \mathrm{ZnO}, \mathrm{C}$, and $\mathrm{SiO}_{2}$ and, therefore, specified four components in the VCA algorithm. This approach proved to be unsuccessful, as it was not possible to separate the $\mathrm{C}$ and $\mathrm{SiO}_{2}$ signals into two separate spectra, with the 3 rd and 4 th components both retaining signatures of $\mathrm{Si}, \mathrm{C}$, and $\mathrm{O}$.

Instead, decomposition into three components proved to be more successful and provided a meaningful physical decomposition of the recorded signal. The resulting three spectral components are shown in Fig. 3. The $\mathrm{Si}$ and $\mathrm{ZnO}$ contributions are separated clearly from each other, with the $\mathrm{Si}$ component retaining only the $\mathrm{Si} \mathrm{K}$ and $\mathrm{Si} \mathrm{L}$ edges, the $\mathrm{ZnO}$ component retaining only the $\mathrm{Zn} \mathrm{L}$ and $\mathrm{O} \mathrm{K}$ edges, and the third component containing $\mathrm{Si}, \mathrm{C}$, and $\mathrm{O}$ peaks. The spectral contributions to the $\mathrm{O} \mathrm{K}$ edge are enlarged in the lower left of Fig. 3 and demonstrate fingerprints that have previously been shown to correspond to $\mathrm{ZnO}$ (Ref. 16) and $\mathrm{SiO}_{2}$ (Ref. 11) (marked A and B, respectively). Interestingly, the Si component does not contain a contribution from the $\mathrm{O} \mathrm{K}$ edge, indicating that two distinct types of bonding to $\mathrm{Si}$ atoms have been separated from each other using VCA, one of which is associated only with surrounding $\mathrm{Si}$ atoms and the other with $\mathrm{Si}, \mathrm{O}$, and $\mathrm{C}$ atoms. $\mathrm{Si}, \mathrm{C}$, and $\mathrm{O}$ are not likely to form a single alloy, but could not be separated from each other using VCA in the present study, as they are always present together in the spectra. Nevertheless, it is most likely that they are present in the form of $\mathrm{SiO}_{2}$ and $\mathrm{C}$. The spectral contributions to the $\mathrm{Zn}$ L edge are enlarged in the lower right of Fig. 3 and confirm that all of the $\mathrm{Zn}$ is associated only with $\mathrm{ZnO}$. 

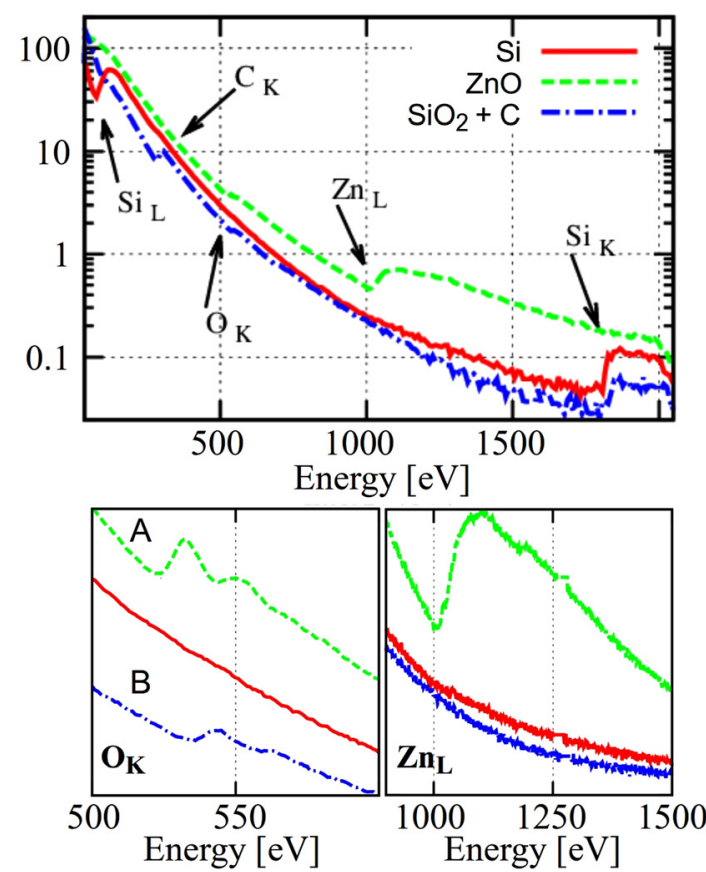

FIG. 3. Spectral components obtained using vertex component analysis from the EEL spectrum image signals shown in Fig. 2. The regions of the spectra close to the $\mathrm{O} \mathrm{K}$ and $\mathrm{Zn} \mathrm{L}$ edges are enlarged in the lower graphs. The edges of the different elements are marked using arrows in the top graph. A and $\mathrm{B}$ refer to $\mathrm{O} \mathrm{K}$ edge signals extracted for the $\mathrm{ZnO}$ and $\mathrm{SiO}_{2}$ components, respectively.

Figure 4 shows final "chemical" maps derived using the spectral decomposition shown in Fig. 3. The Si map now shows only the intensity of the pure Si component and does not contain an $\mathrm{SiO}_{2}$ contribution, in contrast to Fig. 2. The $\mathrm{ZnO}$ map is related closely to the $\mathrm{Zn}$ and $\mathrm{O}$ maps shown in Fig. 2. The $\mathrm{SiO}_{2}+\mathrm{C}$ map contains a measurable signal across the entire specimen and is likely to be due to TEM sample contamination during TEM sample preparation and/ or during the TEM experiment. In this way, SU allows $\mathrm{O}$ in the $\mathrm{ZnO}$ in the upper part of the defect to be distinguished from $\mathrm{O}$ in $\mathrm{SiO}_{2}$ in the lower part.

Our results show that STEM spectrum imaging can be combined with VCA post-processing to characterise a stateof-the-art $\mu \mathrm{c}-\mathrm{Si}: \mathrm{H}$ solar cell and to demonstrate that $\mathrm{ZnO}$ deposited using LP-CVD can creep into a defective region, most probably during deposition of the top contact of the device. The fact that such diffusion of $\mathrm{ZnO}$ is possible indicates that the defective regions are primarily empty following growth of the active $\mu \mathrm{c}-\mathrm{Si}: \mathrm{H}$ layer, at least close to the front contact. Given that EDX and EEL spectroscopy, as applied in the present study, have detection limits of only $\sim 0.1$ at. $\%$ at best, it is conceivable that some $\mathrm{ZnO}$ may have diffused through the empty defective area as far as the bottom contact, resulting in the possibility of a short-circuit of the solar cell. The use of a higher-solid-angle EDX detector or cumulative acquisition of EEL spectra in a future study may allow lower concentrations of $\mathrm{ZnO}$ to be mapped in such devices. Significantly, our combined analysis techniques allow true "chemical" rather than "elemental" mapping and provide a

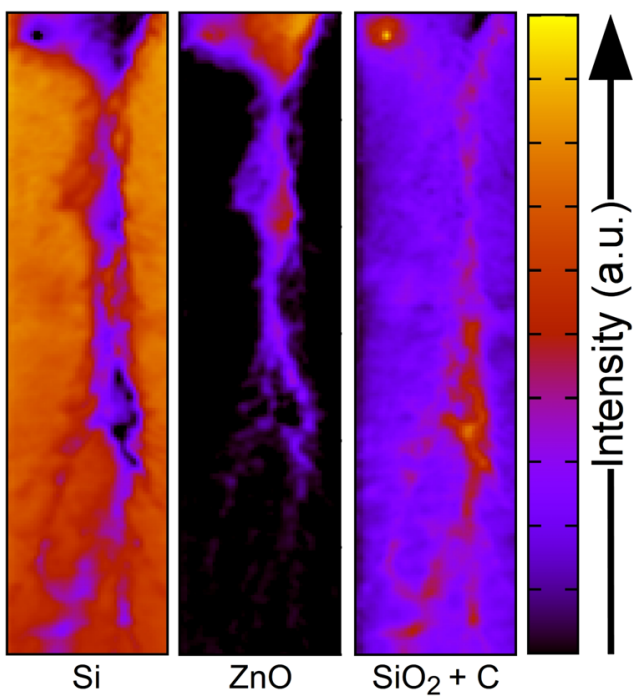

FIG. 4. Maps of the three spectral components obtained using vertex component analysis from the EEL spectrum image signals shown in Fig. 2, corresponding to the spectral signatures shown in Fig. 3. The field of view in each image is $0.31 \mu \mathrm{m} \times 1.34 \mu \mathrm{m}$.

better understanding of the true natures of defective regions in solar cells, paving the way towards the growth of devices with improved efficiencies.

The authors acknowledge financial support from the European Union under the Seventh Framework Programme under a contract for Energy (Reference 241477-SiliconLight) and under a contract for an Integrated Infrastructure Initiative (Reference 312483-ESTEEM2).

${ }^{1}$ H. Iida, N. Shiba, T. Mishuku, H. Karasawa, A. Ito, and Y. Hayashi, IEEE Electron Device Lett. 4, 157 (1983).

${ }^{2}$ T. Söderström, F.-J. Haug, X. Niquille, and C. Ballif, Prog. Photovoltaics 17, 165 (2009).

${ }^{3}$ N. Senoussaoui, M. Krause, J. Müller, E. Bunte, T. Bammer, and H. Stiebig, Thin Solid Films 451, 397 (2004).

${ }^{4}$ C. Haase and H. Stiebig, Prog. Photovoltaics 14, 629 (2006).

${ }^{5}$ M. Python, O. Madani, D. Dominé, F. Meillaud, E. Vallat-Sauvain, and C. Ballif, Sol. Energy Mater. Sol. Cells 93, 1714 (2009).

${ }^{6}$ Y. Nasuno, M. Kondo, and A. Matsuda, Jpn. J. Appl. Phys., Part 1 41, 5912 (2002).

${ }^{7}$ S. Hänni, D. T. L. Alexander, L. Ding, G. Bugnon, M. Boccard, C. Battaglia, P. Cuony, J. Escarré, G. Parascandolo, S. Nicolay, M. Cantoni, M. Despeisse, F. Meillaud, and C. Ballif, IEEE J. Photovoltaics 3, 11 (2013).

${ }^{8}$ A. Čampa, J. Krč, and M. Topič, J. Appl. Phys. 105, 083107 (2009).

${ }^{9}$ K. Suenaga, M. Tence, C. Mory, C. Colliex, H. Kato, T. Okazaki, H. Shinohara, K. Hirahara, S. Bandow, and S. Iijima, Science 290, 2280 (2000).

${ }^{10}$ M. Duchamp, C. B. Boothroyd, M. S. Moreno, B. B. van Aken, W. J. Soppe, and R. E. Dunin-Borkowski, J. Appl. Phys. 113, 093513 (2013).

${ }^{11}$ P. E. Batson, K. L. Kavanagh, C. Y. Wong, and J. M. Woodall, Ultramicroscopy 22, 89 (1987).

${ }^{12}$ N. Dobigeon and N. Brun, Ultramicroscopy 120, 25 (2012).

${ }^{13}$ N. Bonnet, N. Brun, and C. Colliex, Ultramicroscopy 77, 97 (1999).

${ }^{14}$ F. De la Penã, M.-H. Berger, J.-F. Hochepied, F. Dynys, O. Stephan, and M. Walls, Ultramicroscopy 111, 169 (2011).

${ }^{15}$ J. Nascimento and J. Dias, IEEE Trans. Geosci. Remote Sens. 43, 898 (2005).

${ }^{16}$ M. Heidelmann, J. Barthel, and L. Houben, Ultramicroscopy 109, 1447 (2009). 